\title{
Impact of CT slice thickness on volume and dose evaluation during thoracic cancer radiotherapy
}

This article was published in the following Dove Press journal:

Cancer Management and Research

\section{Huanli Luo \\ Yanan He \\ Fu Jin \\ Dingyi Yang \\ Xianfeng Liu \\ Xueqi Ran \\ Ying Wang}

Department of Radiation Oncology, Chongqing University Cancer Hospital

\& Chongqing Cancer Institute

\& Chongqing Cancer Hospital,

Chongqing, 400030, China
Correspondence: $\mathrm{Fu}$ Jin

Department of Radiation Oncology, Chongqing University Cancer Hospital \& Chongqing Cancer Institute \& Chongqing Cancer Hospital, No. I8I Hanyu Road, Shapingba District, Chongqing 400030, China

Tel $+86236530 \quad 1317$

Fax +8623653I 0296

Email jfazj@।26.com
Introduction: Accurate delineation of targets and organs at risk (OAR) is required to ensure treatment efficacy and minimize risk of normal tissue toxicity with radiotherapy. Therefore, we evaluated the impacts of computed tomography (CT) slice thickness and reconstruction methods on the volume and dose evaluations of targets and OAR.

Patients and methods: Eleven CT datasets from patients with thoracic cancer were included. $3 \mathrm{D}$ images with a slice thickness of $2 \mathrm{~mm}(2-\mathrm{CT})$ were created automatically. Images of other slice thickness (4-CT, 6-CT, 8-CT, 10-CT) were reconstructed manually by the selected 2D images using two methods; internal tumor information and external CT Reference markers. Structures and plans on 2-CT images, as a reference data, were copied to the reconstructed images. Results: The maximum error of volume was $84.6 \%$ for the smallest target in $10-\mathrm{CT}$, and the maximum error $\left(\geq 20 \mathrm{~cm}^{3}\right)$ was $10.1 \%, 14.8 \%$ for the two reconstruction methods, internal tumor information and external CT Reference, respectively. Changes in conformity index for a target of $<20 \mathrm{~cm}^{3}$ were $5.4 \%$ and $17.5 \%$ in $8-\mathrm{CT}$. Changes on $\mathrm{V}_{30}$ and $\mathrm{V}_{40}$ of the heart were considerable. In the internal tumor information method, volumes of hearts decreased by $3.2 \%$ in 6-CT, while $\mathrm{V}_{30}$ and $\mathrm{V}_{40}$ increased by $18.4 \%$ and $46.6 \%$.

Conclusion: The image reconstruction method by internal tumor information was less affected by slice thickness than the image reconstruction method by external CT Reference markers. This study suggested that before positioning scanning, the largest section through the target should be determined and the optimal slice thickness should be estimated.

Keywords: computed tomography, slice thickness, thoracic cancer, dose, image reconstruction

\section{Introduction}

The accurate delineation of targets and organs at risk (OAR) is crucial for radiotherapy (RT). Despite the rapid development of image techniques, such as MRI and PET/CT, improving the accuracy of target definitions, ${ }^{1-3}$ computed tomography $(\mathrm{CT})$ imaging is still the main medical modality in defining targets and OAR for RT.

The slice thickness of a CT image is one of the important factors influencing the definition of targets and OAR. ${ }^{4}$ Based on a water phantom, Srivastava et al found that the variability of volumes with slice thicknesses was considerable, especially for small volume structures such as $1-3 \mathrm{~cm}^{3}$, and the differences were smaller for larger volumes with a cutoff of about $20 \mathrm{~cm}^{3} .^{5}$ Our previous phantom studies, based on a series of spherical phantoms ranging from 0.02 to $1151 \mathrm{~cm}^{3}$ with slice thicknesses of 1.0, 1.5, 2.0, 2.5, 3.0, 4.0, 5.0, 7.5 and $8.0 \mathrm{~mm}$, showed that the volume of $<1 \mathrm{~cm}^{3}$ was underestimated $2 \%-100 \%$ for a slice thickness of $\leq 5 \mathrm{~mm}$, but overestimated up 
to $112 \%$ for a slice thickness $>5 \mathrm{~mm}$ for volumes of $>1 \mathrm{~cm}^{3}$. The relative error decreased, and it was nearly zero for a slice thickness $>100 \mathrm{~cm}^{3}$. ${ }^{6}$ Prionas et al also performed a similar study and showed that a smaller slice thickness and a larger volume produced a more accurate volume assessment than larger slice thicknesses and smaller volumes. ${ }^{7}$

The error on the definition of targets and OAR ultimately affects dose evaluation and comparison of treatment plans, since the dose-volume histogram (DVH) relies on computation of doses to certain volumes in a treatment planning system (TPS). ${ }^{8,9}$ Some radiotherapy technologies employing high gradients of dose, such as intensity-modulated radiotherapy (IMRT) and stereotactic body radiotherapy, are more sensitive to these errors, compared to three-dimensional conformal radiation therapy. Some studies demonstrated that the insufficient delineation of a planning target volume because of target mobility was a contributing factor in local failure after curative radiotherapy of lung cancer. ${ }^{10,11}$ Similarly, the underestimate or overestimate of targets and OAR caused by different slice thicknesses also affected the treatment efficacy and the quality of life of the patient. For brain tumors, Prabhakar et $\mathrm{al}^{12}$ suggested that a $2.5 \mathrm{~mm}$ slice thickness was optimum for tumors $<25 \mathrm{~cm}^{3}$ and $5.0 \mathrm{~mm}$ was optimum for tumors $>25$ $\mathrm{cm}^{3}$ in three-dimensional conformal radiation therapy. A study by Caivano et al study recommended 1 or $2 \mathrm{~mm}$ slice thicknesses for small targets in stereotactic body radiotherapy, and 4-6 $\mathrm{mm}$ for other volumes in three-dimensional conformal radiation therapy or IMRT. ${ }^{13} \mathrm{~A}$ similar study was also found on rectal cancer, ${ }^{14}$ however, a study evaluating slice thicknesses in thoracic cancer was not found.

The purpose of our study is to investigate impacts of CT slice thickness and reconstruction methods on volume evaluation and dosimetric coverage of targets and OAR in IMRT from patients with thoracic cancer, to ensure treatment efficacy and minimize risk of normal tissue toxicity with RT.

\section{Patients and methods}

\section{Patient selection}

Eleven patients diagnosed with thoracic cancer were randomly recruited for this trial. Ethics approval for this study was obtained from Chongqing Cancer Hospital's ethics committee, and all patients gave written informed consent. Five of the patients presented with Stage III lung cancer, 4 presented with Stage II and III breast cancer and 2 with Stage II and III esophageal cancer. Their mean age was $55.9 \pm 8.9$ years old and their mean BMI was $23.1 \pm 5.2 \mathrm{~kg} / \mathrm{m}^{2}$.
For each patient, a CT image with a slice thickness of 2 mm (2-CT) was obtained with the patient breathing freely by a CT simulator (Brilliance-16, Philips Medical Systems Inc., Cleveland, OH, USA), while patient was immobilized with thermoplastic fixation, to reduce any error introduced by chest wall motion. Then the 2-CT image was transferred to a commercial TPS (Eclipse version 11, Varian Medical Systems Inc., Palo Alto, CA, USA) through the Digital Imaging and Communications in Medicine network. The senior radiation oncologist delineated the targets and OAR in the 2-CT images. To accurately analyze the impact of slice thickness on volume, the gross tumor volume, clinical target volume, and planning target volume were collectively called target volumes (TVs) in the data analysis of this study. The IMRT plans were developed in the 2-CT image with 6-MV photon beams from a linear accelerator (Varian Clinac iX, Varian Medical Systems Inc., Palo Alto, CA, USA). The plan developed in the 2-CT image met the requirement that $95 \%$ of prescription doses covered at least $100 \%$ of the planning target volumes with acceptable maximum doses.

\section{Image reconstruction}

In general, the three-dimensional (3D) images ware created from multiple two-dimensional (2D) images either automatically (using all the patient's 2D images) or manually (using a range of manually selected 2D images) in accordance with the primary axes of the $2 \mathrm{D}$ images. In this study, the 2-CT 3D images ware created automatically using all the patient's 2D images; 3D images with slice thicknesses of 4, 6, 8, and $10 \mathrm{~mm}$ (4-CT, 6-CT, 8-CT, and 10-CT) were reconstructed manually in the selection workspace of Eclipse TPS using a range of manually selected $2 \mathrm{D}$ images. When selecting $2 \mathrm{D}$ images manually, two methods were adopted; the internal tumor information method and the external CT Reference three markers method. In the internal tumor information method, the layer showing the largest section through the target was selected as the initial layer (Figure 1A) and then the alternate selection at a regular spacing up and down was performed. In the external the CT Reference markers method, the layer showing the surface three metal markers was selected as the initial layer (Figure 1B) and then the alternate selection at a regular spacing up and down was performed.

Subsequently, for each series, 4-, 6-, 8-, and 10-CT images were merged with 2-CT image using rigid registration (Offline Review, version 11, Varian Medical Systems Inc., Palo Alto, CA, USA). All the targets and OAR delineated 


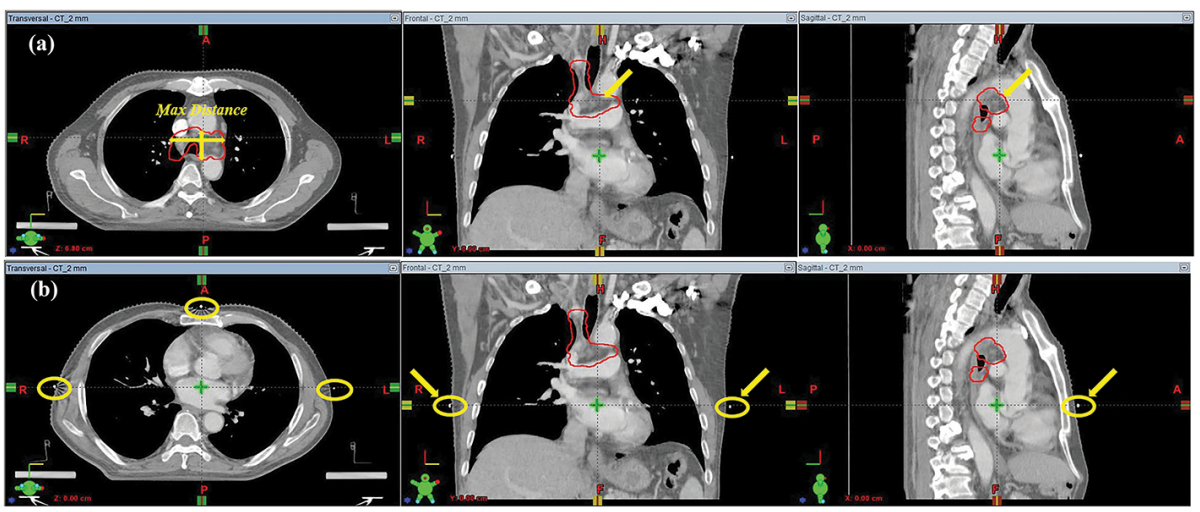

Figure I Selection of the initial layer, when reconstructing 3D images with slice thickness of $4,6,8$, and $10 \mathrm{~mm}$.

Notes: (A) Internal tumor information method; the layer showing the largest section through the target; (B) external CT Reference markers method; the layer showing three metal markers.

Abbreviation: $\mathrm{CT}$, computed tomography.

in the 2-CT image were copied, slice by slice, to the reconstructed images to avoid the delineating deviation caused by intra-observer variability. The treatment plans were also copied to the reconstructed images and computed independently.

\section{Plan evaluation}

A conformity index, defined in Equation 1, ${ }^{15,16}$ was used to measure the target dose conformity.

$$
C I=\frac{T V_{R I}}{T V} \cdot \frac{T V_{R I}}{V_{R I}}
$$

where, $T V_{\mathrm{RI}}$ was the target volume covered by the reference isodose $(95 \%), V_{\mathrm{RI}}$ was the volume covered by the reference isodose (95\%), and $T V$ was the target volume. The target dose homogeneity was measured with a homogeneity index (HI) defined in Equation 2. ${ }^{17}$

$$
H I=\frac{D_{5 \%}-D_{95 \%}}{D_{\text {mean }}}
$$

where, $D_{5 \%}, D_{95 \%}$ indicated that at least $5 \%, 95 \%$ of the target volume received this dose, and $D_{\text {mean }}$ was the mean dose to target. The HI value was between 0 and 1 , with 0 representing the ideal homogeneity, whereas the CI value was between 0 and 1, with one representing the ideal conformity. In OAR, the minimum and maximum dose of spinal cord, $\mathrm{V}_{30}$ and $\mathrm{V}_{40}$ of heart, and $\mathrm{V}_{20}$ and $\mathrm{V}_{30}$ of lung were analyzed and compared.

\section{Statistical analysis}

Eleven CT series were obtained, and each series included a 2-CT image created automatically and a sub-series of
4-CT, 6-CT, 8-CT, 10-CT images reconstructed manually. For each sub-series, all the data obtained in reconstructed images were compared with that obtained in the corresponding 2-CT image by using SPSS software version 23 (IBM Armonk, NY, USA).

\section{Results \\ Volume analysis}

Thirty-one irregular TVs were analyzed in this study. These targets were grouped, as shown in Table 1. Figure 2 shows all TVs normalized to that in 2-CT images, and Figure 3 shows the statistical variations on volumes with respect to that in 2-CT images.

The TV deviations in both methods decreased with increasing slice thickness of CT image, and the change in internal tumor information method was slightly smaller and smoother than that in the external CT Reference markers method, especially for targets of $<50 \mathrm{~cm}^{3}$. As shown in Figure 2, for targets of $1.3 \mathrm{~cm}^{3}$, the volume deviations in 10-CT, 8 -CT and 6-CT were $84.62 \%, 38.46 \%$ and $15.39 \%$ in internal tumor information method, and $84.62 \%$, $84.62 \%$ and $13.77 \%$ in the external CT Reference markers method.

\section{Dose conformity and homogeneity of target}

The CI in both methods decreased with increasing slice thickness of CT image, and the change in internal tumor information method was slightly smaller and smoother than that in external CT Reference three markers method. As shown in Figure 4, the changes in CI of small targets $(<20$ $\mathrm{cm}^{3}$ ) were considerable, $5.4 \%, 17.5 \%$ in $8-\mathrm{CT}$, and $6.1 \%$, 
Table I Groups of thirty-one irregular target volumes from eleven patients with thoracic cancer

\begin{tabular}{|c|c|c|c|c|c|c|}
\hline Groups $/ \mathrm{cm}^{3}$ & $0-20$ & $20-50$ & $50-100$ & $100-200$ & $200-500$ & $500-1,000$ \\
\hline $\begin{array}{l}\text { Number of } \\
\text { targets }\end{array}$ & 4 & 5 & 4 & 5 & 8 & 5 \\
\hline Volume $/ \mathrm{cm}^{3}$ & $9.8 \pm 6.1$ & $35.1 \pm 6.4$ & $79.2 \pm 9.3$ & $155.1 \pm \mid 7.7$ & $339.4 \pm 103.2$ & $658.3 \pm 75.1$ \\
\hline
\end{tabular}

Abbreviations: $\mathrm{N}$, number of patients; TVs, target volumes.
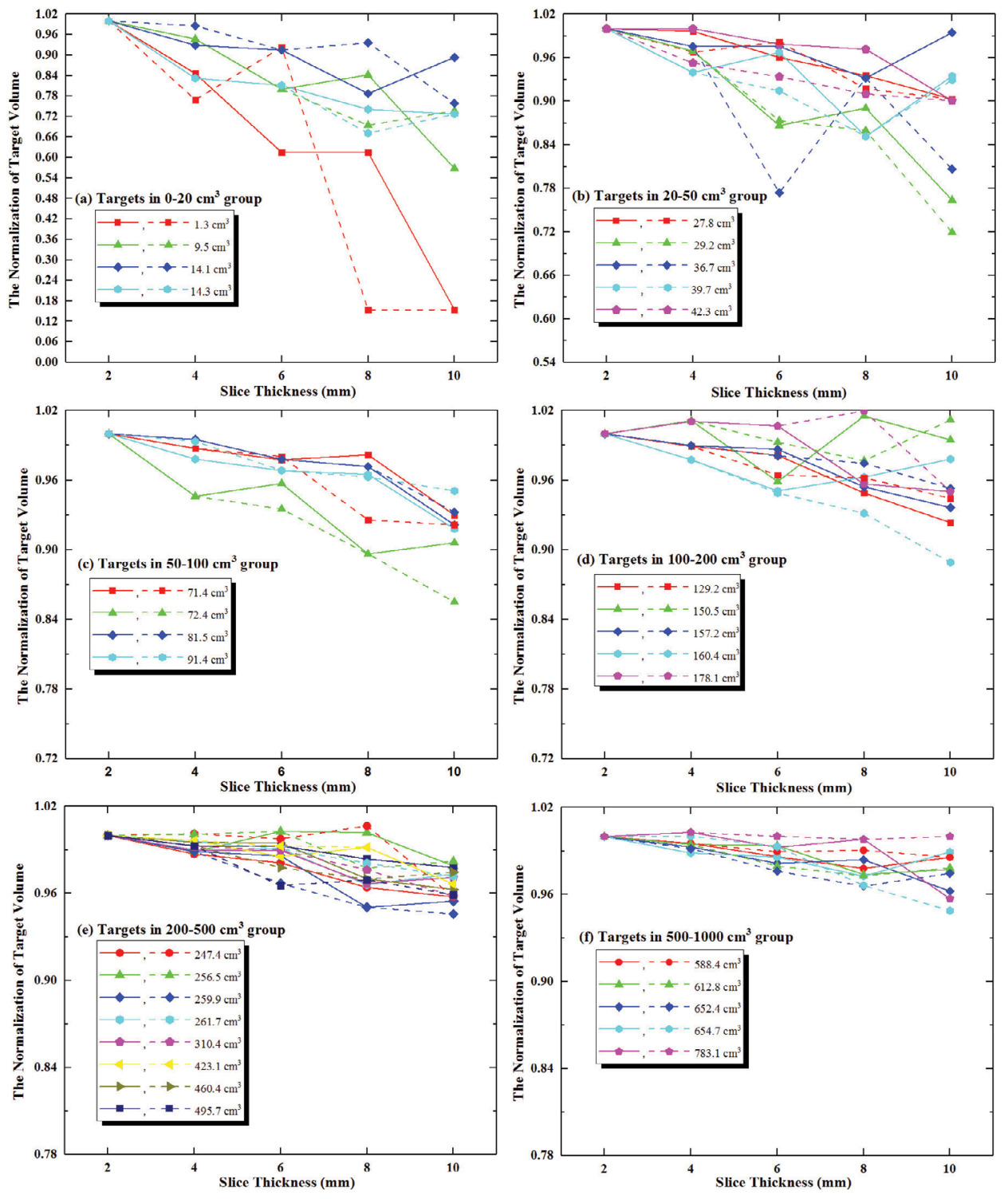

Figure 2 The TVs in all series for both methods, normalized to that in 2-CT images.

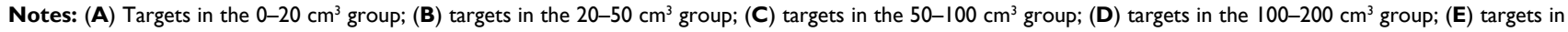
the $200-500 \mathrm{~cm}^{3}$ group; (F) targets in the $500-1,000 \mathrm{~cm}^{3}$ group. Here, the internal tumor information method is represented by the solid line with symbols, and the external CT Reference markers method is represented by the dashed line with symbols.

Abbreviations: CT, computed tomography; TVs, target volumes.

9.2\% in 6-CT, for both methods, respectively; for targets of $\geq 20 \mathrm{~cm}^{3}$, the decrease was $<2.0 \%$ in $6-\mathrm{CT},<4.3 \%$ in $8-\mathrm{CT}$, and $<5.8 \%$ in $10-\mathrm{CT}$.

The change in HI was not sensitive to slice thickness and the difference between both methods was irregular, as shown in Figure 5. For some cases with breast cancer, the change of HI increased with the increase of slice thickness and this value was $\leq 4.67 \%$. The change in HI was $-5.2 \%-6.08 \%$ for lung cancer and $-8.33 \%-8.08 \%$ for esophageal cancer. 


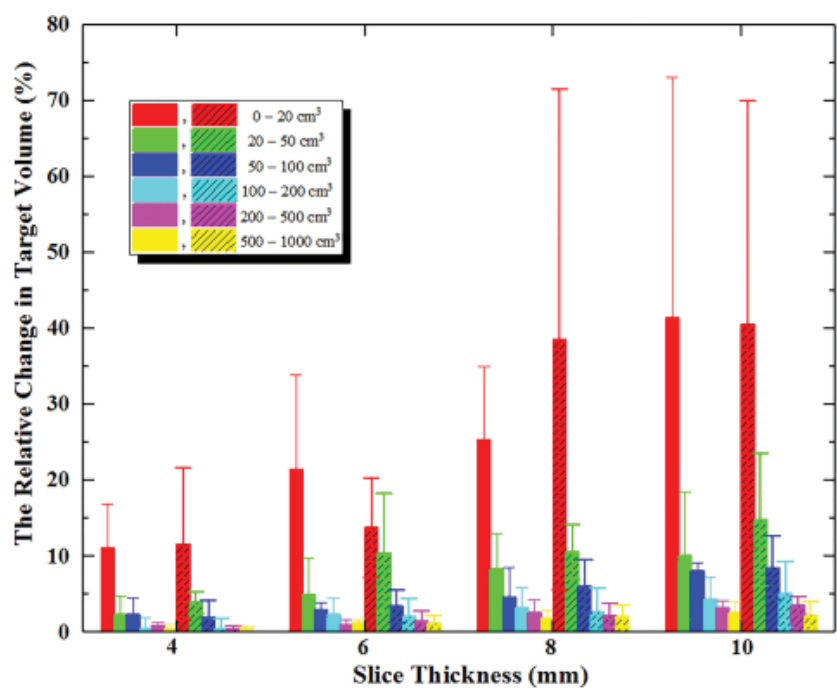

Figure 3 Relative change in TVs with respect to 2-CT images.

Notes: Error bars indicate \pm I SD. Here, the internal tumor information method is represented by vertical column graph, and the external CT Reference markers method is represented by vertical column graph filled by dense oblique lines. Abbreviations: CT, computed tomography; TVs, target volumes.

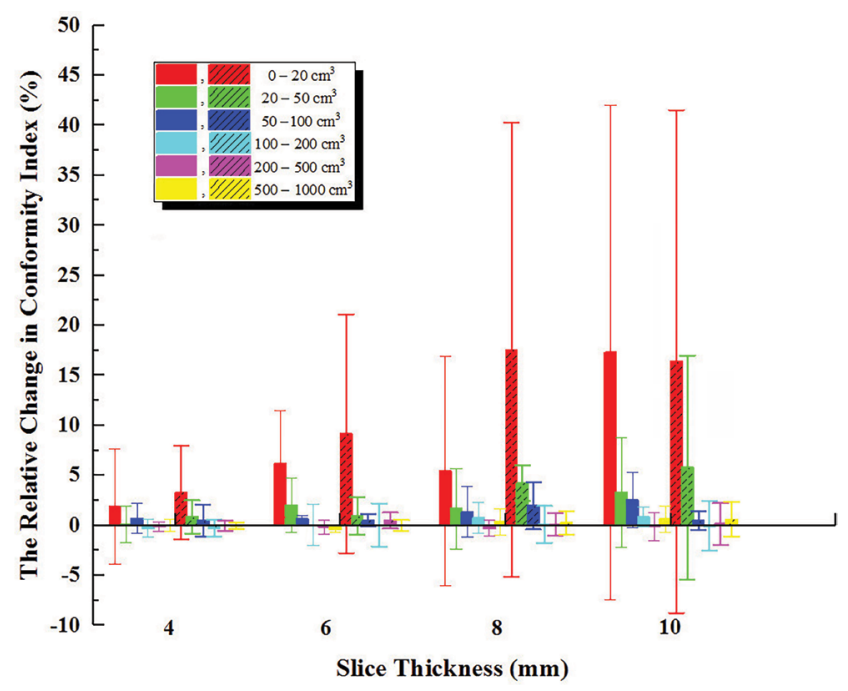

Figure 4 The relative change in target $\mathrm{Cl}$ with respect to 2-CT images. Notes: Error bars indicate \pm I SD Here, the internal tumor information method is represented by vertical column graph, and the external CT Reference markers method is represented by vertical column graph filled by some dense oblique lines. Abbreviations: $\mathrm{Cl}$, conformity index; $\mathrm{CT}$, computed tomography.

\section{OAR sparing}

The volumes of spinal cord, lung and heart were $45.7 \pm 11.5$ $\mathrm{cm}^{3}$ (range of $31.3-62.1 \mathrm{~cm}^{3}$ ), 2834.1 $\pm 860.8 \mathrm{~cm}^{3}$ (range of $1635.0-4285.7 \mathrm{~cm}^{3}$ ) and $584.3 \pm 79.5 \mathrm{~cm}^{3}$ (range of $416.5-722.1 \mathrm{~cm}^{3}$ ) in 2-CT, respectively. The impacts of slice thickness on the minimum dose of spinal cord, and $\mathrm{V}_{30}, \mathrm{~V}_{40}$ of heart were considerable, as shown in Figure 6A, C, D. For example, in the internal tumor information method, the

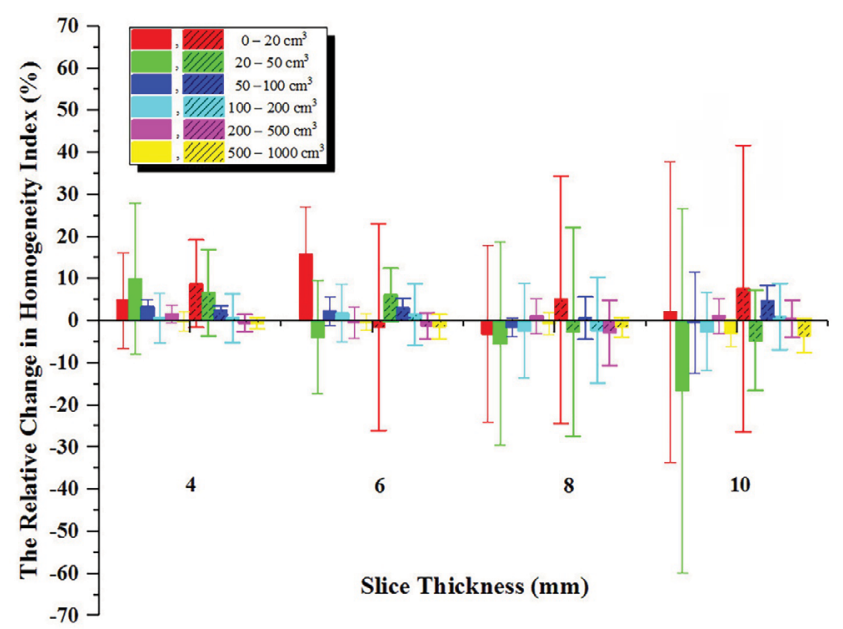

Figure 5 The relative change in target $\mathrm{HI}$ with respect to 2-CT images.

Notes: Error bars indicate \pm I SD. Here, the internal tumor information method is represented by vertical column graph, and the external CT Reference markers method is represented by vertical column graph filled by some dense oblique lines. Abbreviations: $\mathrm{CT}$, computed tomography; $\mathrm{HI}$, homogeneity index.

volume of spinal cord decreased by $7.7 \%$ between $2-\mathrm{CT}$ and $10-\mathrm{CT}$ in Patient 1, while its minimum dose increased by $88.1 \%$; for heart, the volume decreased by $3.2 \%$ between 2-CT and 6-CT, while the $\mathrm{V}_{30}$ and $\mathrm{V}_{40}$ increased by $18.4 \%$ and $46.6 \%$, respectively. The slice thickness had less impact on maximum dose to spinal cord, as shown in Figure 6B. The mean dose of spinal cord increased by an average of $3.1 \%$. The slice thickness had no effect on dose to lung, as shown in Figure 6E, F. The dose to OAR made no significant difference between both methods.

\section{Discussion}

Prior to this study, the Chongqing Cancer Institute carried out a phantom study and proposed a simple and feasible quality assurance method for estimating the accuracy of margin growth. ${ }^{6}$ This study investigated irregular TVs in thoracic cancer patients. A considerable variability of TVs between different CT slice thicknesses, especially for small targets, such as $1.3 \mathrm{~cm}^{3}$ tumors, was found; the variation was smaller for larger targets. Our study showed that CT images with slice thickness of $<4 \mathrm{~mm}$ would be optimum for small targets $(<20$ $\mathrm{cm}^{3}$ ) in IMRT of thoracic cancer patients. However, some studies revealed a noise-limited minimum thickness, such as the slice thickness of $1.2 \mathrm{~mm}$ superior to $0.6 \mathrm{~mm}$ because of increasing dimpling artifacts. ${ }^{18}$ Similarly, the slice thickness with the range of $0.8-2 \mathrm{~mm}$ had no considerable impact on the residual setup error of stereotactic radiosurgery target localization. ${ }^{19}$ In brain cancer, previous studies suggested $2.5 \mathrm{~mm}$ for tumor of $<25 \mathrm{~cm}^{3}$ in three-dimensional conformal radia- 

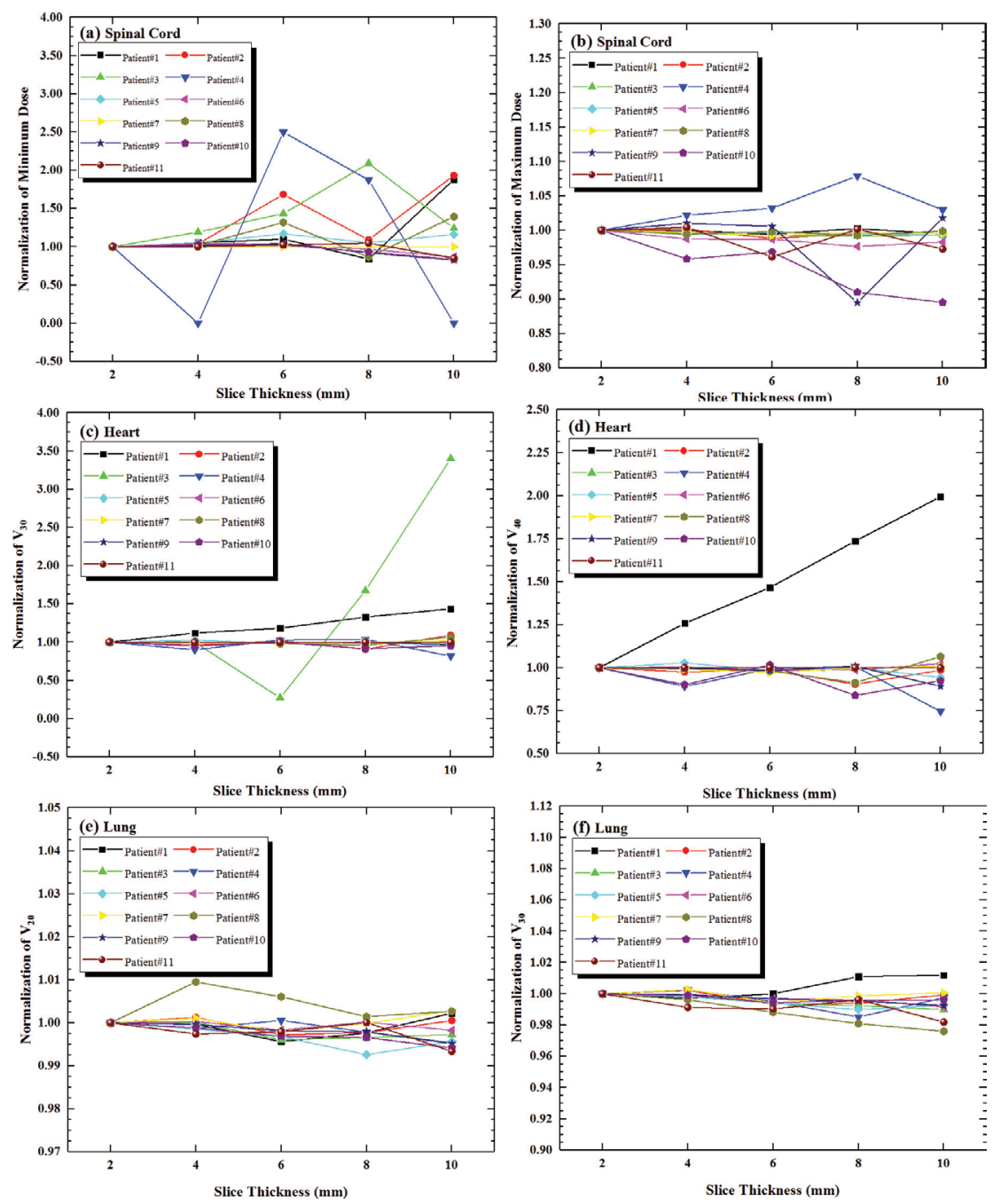

Figure 6 Doses of spinal cord, heart and lung for eleven patients in internal tumor information method, normalized to doses derived from 2-CT images. Notes: (A) Minimum dose in spinal cord; (B) maximum dose in spinal cord; (C) $\bigvee_{30}$ in Heart; (D) $\bigvee_{40}$ in Heart; (E) $\bigvee_{20}$ in Lung; (F) $\bigvee_{30}$ in Lung.

tion therapy and 1 or $2 \mathrm{~mm}$ in stereotactic body radiotherapy with small targets. ${ }^{12,13}$ But, the brain tumors were not in any way affected by respiratory motion, and targets of thoracic cancer were different. So, the minimum optimum thickness still needs to be investigated in future work.

The error in volume evaluation ultimately affected the integrity in plan evaluation as well as other radiobiological plan metrics. Our study showed that the conformity index of $95 \%$ isodose decreased with increasing slice thickness of CT image and HI was not affected. For OAR, the cardiac events were related to both dose and irradiated volume of heart. The mean $\mathrm{V}_{20}, \mathrm{~V}_{30}$ and $\mathrm{V}_{40}$ of the contoured whole heart had a considerable impact on the symptomatic cardiac toxicities..$^{20}$ The incidence of risk increased with a variety of dose parameters. ${ }^{21}$ Our study showed that $\mathrm{V}_{30}$ and $\mathrm{V}_{40}$ of whole heart were affected by slice thickness of the CT image. Thus, the optimal slice thickness was crucial for the accurate evaluation of these parameters, to the preferable OAR sparing.

The image reconstruction method also affected the volume and dose evaluations. In Srivastava's study, CT images with different slice thicknesses $(1,2,3,5,10 \mathrm{~mm})$ were obtained by repeating helical scans on a CT simulator. ${ }^{5}$ In a study by Prionas et al, CT images with greater thicknesses $(1.25,2.50$, $5.0 \mathrm{~mm}$ ) were produced by averaging contiguous thin-slice images $(0.625 \mathrm{~mm}) .{ }^{7}$ Unlike both studies, we reconstructed a series of 3D images with greater thickness in Eclipse TPS selection workspace using a range of manually selected 2D images, which was similar to that found by Caivano et al. ${ }^{13}$ However, how to choose the initial layer in the reconstruction process was one concern noticed in our study. We found that the external CT Reference three markers method has the char- 
acteristics of instability and randomness, while the internal tumor information method could maximize the tumor outline and make the smaller deviation in volume estimations, especially for tumor volumes of $<50 \mathrm{~cm}^{3}$, as well as slightly smaller change in CI. These findings showed a potential improvement in the workflow of scanning CT positioning images to obtain the targets and OAR closest to reality.

In the conventional $\mathrm{CT}$ scan for positioning, radiation oncologist positioned one patient and then placed three markers on surface skin or thermoplastic masks according to in-room lasers; an orthophoria topogram was scanned, followed by determining scan range and parameters; afterwards, spiral scanning was performed. Disadvantages of this method are potentially making three markers placed on the different slices, which can cause difficulty in setting the isocenter in TPS, and to potentially lose partial information on the largest section through the target during image reconstruction. An improvement in the workflow was raised in our study. In the improved workflow, the radiation oncologist determined the largest section through the target by reading the orthophoria topogram and then placed three markers on this area. Afterwards, the optimal slice thickness was estimated by tumor size and scan range, to not only ensure three markers on the same slice but also retain the largest section through the tumor during image reconstruction.

The limitation of this study was that the error introduced from chest wall motion in free breathing was not researched. Even so, it was obvious that the optimal slice thickness was crucial for the accurate evaluation of volumes and doses in targets and OAR of thoracic cancer RT.

\section{Conclusion}

CT slice thickness and reconstruction methods are important factors that influence the accurate definition of targets and OAR in thoracic cancer RT, except in respiratory motion. This study concludes that a CT slice thickness of $<4 \mathrm{~mm}$ is suggested for small targets $\left(<20 \mathrm{~cm}^{3}\right)$ in IMRT of thoracic cancer patients, to ensure treatment efficacy and minimize risk of normal tissue toxicity with RT. The reconstruction method by internal tumor information in TPS shows an advantage over the reconstruction by the external CT Reference markers method. An improved scanning workflow for positioning images is proposed to obtain the targets and OAR closest to the reality.

\section{Acknowledgments}

This work was supported by the National Natural Science Foundation of China (11575038), the Performance
Motivation and Guidance Special Project of Chongqing Science \& Technology Commission (cstc2017jxj1130017), the Innovation Project of Social Undertakings and the Livelihood Security Technology of Chongqing Science \& Technology Commission (cstc2016shms-ztzx10002).

\section{Disclosure}

The authors report no conflicts of interest in this work.

\section{References}

1. Wong KH, Panek R, Bhide SA, Nutting CM, Harrington KJ, Newbold $\mathrm{KL}$. The emerging potential of magnetic resonance imaging in personalizing radiotherapy for head and neck cancer: an oncologist's perspective. Br J Radiol. 2017;90(1071):20160768.

2. Pogson EM, Delaney GP, Ahern V, et al. Comparison of Magnetic Resonance Imaging and Computed Tomography for Breast Target Volume Delineation in Prone and Supine Positions. Int J Radiat Oncol Biol Phys. 2016;96(4):905-912.

3. Konert T, Vogel W, Macmanus MP, et al. PET/CT imaging for target volume delineation in curative intent radiotherapy of non-small cell lung cancer: IAEA consensus report 2014. Radiother Oncol. 2015;116(1):27-34.

4. Beneventi S, Chionne F, Gobbi G, Italiani M, Raymondi C. Quantitative CT tomography for radiotherapy treatment planning: Calibration phantom and sources of error. Radiotherapy and Oncology. 1995; 37(1):S41.

5. Srivastava SP, Cheng CW, das IJ. The effect of slice thickness on target and organs at risk volumes, dosimetric coverage and radiobiological impact in IMRT planning. Clin Transl Oncol. 2016;18(5): 469-479.

6. Wang Y, Jin F, Zhou J, Luo H. A simple method of evaluating margingrowing accuracy in image-guided radiation therapy. $\mathrm{Br} J$ Radiol. 2016;89(1062):20140636.

7. Prionas ND, Ray S, Boone JM. Volume assessment accuracy in computed tomography: a phantom study. J Appl Clin Med Phys. 2010;11(2): 168-180.

8. Oinam AS, Singh L, Shukla A, et al. Dose volume histogram analysis and comparison of different radiobiological models using in-house developed software. J Med Phys. 2011;36(4):220-229.

9. Hernando ML, Marks LB, Bentel GC, et al. Radiation-induced pulmonary toxicity: a dose-volume histogram analysis in 201 patients with lung cancer. Int J Radiat Oncol Biol Phys. 2001;51(3):650-659.

10. Balter JM, Ten Haken RK, Lawrence TS, Lam KL, Robertson JM. Uncertainties in CT-based radiation therapy treatment planning associated with patient breathing. Int J Radiat Oncol Biol Phys. 1996;36(1): $167-174$.

11. van Sörnsen de Koste JR, Lagerwaard FJ, Schuchhard-Schipper RH, et al. Dosimetric consequences of tumor mobility in radiotherapy of stage I non-small cell lung cancer--an analysis of data generated using 'slow' CT scans. Radiother Oncol. 2001;61(1):93-99.

12. Prabhakar R, Ganesh T, Rath GK, et al. Impact of different CT slice thickness on clinical target volume for 3D conformal radiation therapy. Med Dosim . 2009;34(1):36-41.

13. Caivano R, Fiorentino A, Pedicini P, Califano G, Fusco V. The impact of computed tomography slice thickness on the assessment of stereotactic, $3 \mathrm{D}$ conformal and intensity-modulated radiotherapy of brain tumors. Clin Transl Oncol. 2014;16(5):503-508.

14. Olsson C, Thor M, Liu M, et al. Influence of image slice thickness on rectal dose-response relationships following radiotherapy of prostate cancer. Phys Med Biol. 2014;59(14):3749-3759.

15. Paddick I. A simple scoring ratio to index the conformity of radiosurgical treatment plans. Technical note. J Neurosurg. 2000;93 Suppl 3(S3):219-222. 
16. Jin F, Wang Y, Wu YZ. A novel correction factor based on extended volume to complement the conformity index. Br J Radiol. 2012;85(1016): e523-e529.

17. Claus F, Mijnheer B, Rasch C, et al. Report of a study on IMRT planning strategies for ethmoid sinus cancer. Strahlenther Onkol. 2002;178(10):572-576.

18. Lalondrelle S, Sohaib SA, Castellano IA, Mears D, Huddart R, Khoo V. Investigating the relationship between virtual cystoscopy image quality and CT slice thickness. Br J Radiol. 2012;85(1016): 1112-1117.
19. Briceno J, Li H, Huang Y, Wen N, Chetty I, et al. SU-E-J-30: The Effect of Planning CT Slice Thickness On Evaluation of SRS Target Localization Accuracy. Med Phys. 2015;42(6):3270.

20. Konski A, Li T, Christensen M, et al. Symptomatic cardiac toxicity is predicted by dosimetric and patient factors rather than changes in $18 \mathrm{~F}$ FDG PET determination of myocardial activity after chemoradiotherapy for esophageal cancer. Radiother Oncol. 2012;104(1):72-77.

21. Wei X, Liu HH, Tucker SL, et al. Risk factors for pericardial effusion in inoperable esophageal cancer patients treated with definitive chemoradiation therapy. Int J Radiat Oncol Biol Phys. 2008;70(3):707-714.
Cancer Management and Research

\section{Publish your work in this journal}

Cancer Management and Research is an international, peer-reviewed open access journal focusing on cancer research and the optimal use of preventative and integrated treatment interventions to achieve improved outcomes, enhanced survival and quality of life for the cancer patient. The manuscript management system is completely online and includes
Dovepress

a very quick and fair peer-review system, which is all easy to use. Visit http://www.dovepress.com/testimonials.php to read real quotes from published authors. 